www. revis tad y o. com

\title{
Disposición a pagar por atributos de Responsabilidad Social Empresaria. Un estudio aplicado a consumidores en el Gran La Plata
}

Willingness to Pay for Attributes of Corporate Social Responsibility. A study applied to consumers in the Gran La Plata

Estefanía Solari

Instituto de Investigaciones Administrativas. Facultad de Ciencias Económicas. Universidad Nacional de La Plata - Argentina estefania.solari@econo.unlp.edu.ar; estesolari@yahoo.com.ar

Fecha de recepción: 24-09-2014

Fecha de aceptación: 22-02-2016

Resumen: El presente trabajo tiene como objetivo determinar la disposición a pagar (DAP) de los consumidores en el Gran La Plata (Buenos Aires) por atributos de Responsabilidad Social Empresaria, así como también analizar si el género, la edad y el contar con nivel educativo universitario completo influye sobre la misma. Como metodología se procedió a estimar modelos de regresión logit y tobit. Del análisis de los resultados se puede destacar, que los encuestados presentan una DAP alta para el atributo de RSE Protección al Medio Ambiente, y que la DAP positiva y su monto se relaciona inversamente con el género y la edad, lo que implica que el monto de la DAP disminuye en caso de ser hombre y a medida que aumenta la edad.

Palabras clave: Responsabilidad Social Empresaria, Disposición a pagar, Protección medio ambiente, logit, tobit.

Summary: This paper aims to determine the willingness to pay (WTP) of consumers in the Gran La Plata (Buenos Aires) by attributes of Corporate Social Responsibility, as well as to analyze if the gender, age and to have a university education full influence the WTP. As methodology it was estimated logit and tobit regression. The analysis of the results can be noted that the respondents have a high WTP for attribute CSR Environmental Protection, and the positive DAP and its amount is inversely related to gender and age, which implies that the amount WTP decreases in case of being man and with increasing age.

Keywords: Corporate Social Responsibility, Willingness to pay, environment protection, logit, tobit.

\section{Introducción}

Las empresas tienen una estrecha interacción con la sociedad, y es así como sus operaciones y actividades influyen en los inversores, el personal, los consumidores, las comunidades y el medio ambiente de varias maneras. Además, en el Siglo XXI los consumidores tienen mayores expectativas puestas en las empresas, y esperan que éstas no sólo obtengan una ganancia para sus inversores, sino que también tengan un accionar honesto y socialmente responsable. A su vez, las empresas no sólo tienen que garantizar la seguridad del lugar de trabajo para sus empleados, sino también satisfacer las demandas económicas y sociales de los mismos. Externamente, las empresas deben tener en cuenta los derechos de los consumidores, y al mismo tiempo ser responsable con actividades como el reciclaje de los recursos y la protección del medio ambiente. En este contexto, los consumidores quieren que las empresas no sólo sean rentables, sino también puedan proporcionar un servicio social (V. Kreng \& M. Huang 2011).
Lo que aún no está analizado es si existe disposición a pagar (DAP) de los consumidores locales por atributos de Responsabilidad Social Empresaria, de manera tal que los empresarios cuenten con información acerca de si podrán invertir en atributos de RSE manteniendo al mismo tiempo su eficiencia.

El objetivo general de la investigación consiste en determinar la disposición a pagar (DAP) de los consumidores en el Gran La Plata por atributos de Responsabilidad Social Empresaria. La importancia de la investigación radica en que no hay estudios sobre la disposición a pagar de los consumidores en el Gran La Plata por atributos de Responsabilidad Social Empresaria, por lo cuál se generará mediante este estudio información útil para la toma de decisiones de los empresarios de la región, en la medida que aporta evidencia que ayude a integrar el objetivo económico de las empresas con las obligaciones éticas y sociales propias de Responsabilidad Social Empresaria. A su vez, el desarrollo y prueba de la metodología seleccionada, así como el análisis posterior de los resultados, podrá ser replicado sobre consumidores de otros lugares con 
el fin de analizar la DAP de otros consumidores, así como también realizar estudios comparativos que resulten relevantes.

El presente estudio se estructura de la siguiente manera, el primer apartado consiste en una revisión bibliográfica, luego se definen los objetivos detallados de la investigación, a continuación se describe la metodología seleccionada y se exponen los resultados y por último se reflexiona sobre las conclusiones más relevantes de la investigación.

\section{Revisión bibliográfica}

De la revisión de la bibliografía sobre RSE, surgen diversas definiciones, por eso cabe reafirmar que en este trabajo se considera el concepto de RSE, creyéndola no como "dádiva" que hacen las empresas a la comunidad en la que operan, sino como un concepto mucho más amplio, considerando que las obligaciones de la empresa son más amplias y se deberían tener en cuenta todas aquellas personas y grupos que se "juegan" algo con la actuación de la empresa- y que pueden potencialmente verse afectadas por ella todos los grupos de interés aunque no tengan relaciones contractuales con la misma.

No debe confundirse acción social con RSE, simplemente "porque una empresa puede ser socialmente responsable sin dar ni un euro para buenas causas" Lozano (2003).

De esta manera la RSE no debe limitarse a una mera operación de maquillaje siguiendo una moda sino que las empresas deben integrar en su estrategia los principios propios de la RSE y tener en cuenta el impacto que sus decisiones tienen sobre empleados, proveedores, clientes y, en suma, sus repercusiones sobre el medio ambiente y la sociedad (Fernández de Castro Rivera, 2008).

Ahora se considera adecuada la definición sobre RSE mencionada anteriormente, aclarando que tomar en cuenta los intereses de los mencionados grupos de interés no implica relegar a los accionistas ya que esta en el propio interés de estos.

El tema de la RSE ha ido tomando cada vez mayor dimensión, es así como la International Organization for Standardization, ha escrito una Guía sobre responsabilidad social (ISO 26000:2010), es importante realizar una breve reseña acerca del aporte que realiza esta norma sobre el tema analizado, la misma pretende ayudar a las organizaciones a contribuir al desarrollo sostenible. Tiene como propósito fomentar que las or- ganizaciones vayan más allá del cumplimiento legal, reconociendo que el cumplimiento de la ley es una obligación fundamental para cualquier organización y una parte esencial de su responsabilidad social. Se pretende promover un entendimiento común en el campo de la responsabilidad social y complementar otros instrumentos e iniciativas relacionadas con la responsabilidad social, sin reemplazarlos.

Es importante la aclaración que hace la norma ISO 26000 sobre el rol del Estado: "esta Norma, no puede reemplazar, transformar o modificar de ninguna forma el deber del Estado de actuar por el interés público. Esta Norma Internacional no proporciona una orientación sobre qué es lo que debería someterse a regulación jurídica obligatoria; tampoco pretende abordar cuestiones que sólo pueden resolverse apropiadamente a través de las instituciones políticas. El Estado es el único con poder de crear y hacer cumplir las leyes, lo que le hace diferente a las organizaciones... El rol del Estado es esencial para garantizar la aplicación efectiva de las leyes y regulaciones, con el fin de fomentar una cultura de cumplimiento de la ley".

\subsection{RSE de la teoría a la práctica}

Si las prácticas de RSE no redundan en ciertas ventajas para aquellos que las aplican las empresas consideraran que la RSE es mera filantropía o, lo que es peor, simplemente una operación de maquillaje.

Es importante mencionar el aporte que realiza Fernández de Castro Rivera (2008), el mismo plantea que existe un dilema del prisionero cuando se analiza la aplicación de los principios de la RSE si no se pueden encontrar razones teóricas que justifiquen que los mismos incrementan la eficiencia de las empresas. Así continúa que si este fuera el caso y conllevasen una pérdida de eficiencia, o en el caso más favorable fuesen neutrales, una empresa estaría mejor si no los aplica- al ahorrarse los costes correspondientesmientras que sus competidoras se comprometen con los mismos. Por el contrario, si todos siguen el ejemplo de aquel que siguió un comportamiento oportunista el mundo seria un lugar más desagradable para vivir. Por último establece, que el problema se resolvería si efectivamente hubiera una correlación entre la RSE y la eficiencia. En tal caso, no habría nada que ganar si alguien decidiese permanecer al margen de la RSE y, obviamente, no lo haría al no haber los incentivos adecuados para comportarse de ese modo.

Las corporaciones deberían analizar las acciones de RSE, no como un costo o una obra de caridad, sino teniendo en cuenta que las mismas pueden ser una 
fuente de oportunidades, innovación y ventaja competitiva (Porter y Kramer, 2006)

\subsection{Algunos antecedentes bibliográfi- cos sobre disposición a pagar por atributos de Responsabilidad Social Empresaria}

De la revisión de la bibliografía surgieron algunas investigaciones que aportan valiosa información sobre el tema:

Fernández Kraz y Merino Castello (2005), enuncian que algunos autores, han analizado las características de la demanda socialmente responsable a partir de un estudio de opinión realizados a la población, es decir, preguntando directamente a los ciudadanos. Dichos trabajos se centran en la opinión y valoración general de los ciudadanos, sin embargo, pocos de ellos intentan cuantificar la disposición a pagar por las distintas conductas socialmente responsables. Establecen a su vez, que la mayoría obtienen como resultados que los consumidores valoran positivamente las actuaciones de RSE, especialmente aquellas orientadas a proteger el medio ambiente o los derechos de los trabajadores, así como las empresas que las llevan a cabo.

Por otro lado, Mohr y Webb (2005) (citado por Feldman P. \& Reficco E. ,2012) encuentran para EE.UU. que la RSE tiene un impacto positivo sobre la valuación de la empresa y la intención de compra, y, además, que "la valoración que tienen los consumidores de la RSE es utilizada como un criterio de compra, incluso en situaciones en las que no hay paridad competitiva en los productos". Del mismo modo, Miles y Munilla (2004) (citado por Feldman P. \& Reficco E. ,2012), sugieren que los consumidores estarían dispuestos a pagar un sobreprecio por los productos de empresas que tienen un trato socialmente responsable hacia sus trabajadores.

Arrendo Trapero et al (2010), en un artículo sobre RSE establece como objetivo del mismo, demostrar que un consumidor socialmente responsable está interesado en informarse sobre la Responsabilidad Social Empresaria (RSE) de la empresa así como está dispuesto a pagar un precio más alto por aquel producto que ha sido realizado de forma socialmente responsable. Como resultado de la investigación realizada a estudiantes de pregrado de una universidad privada en México, los autores concluyeron que la mayoría de los consumidores mexicanos sigue guiando su decisión de compra tomado como criterio el precio y la mayor parte de ellos no están interesados en ser informados sobre las prácticas de RSE.
Por otra parte, es importante destacar la publicación sobre RSE realizada en el año 2010 por la Confederación de Consumidores y Usuarios (CECU) de España, la misma se llevo a cabo en el ámbito de España, en individuos de más de 18 años de edad, obteniéndose una muestra de 1.004 entrevistas. Es de destacar los resultados de una de sus preguntas, en la misma se indagaba sobre lo siguiente: "Si tuviera información, al comprar un producto o contratar un servicio, ¿elegiría a la empresa con mejor comportamiento social o medioambiental, aunque esta opción resultara más cara?", de sus respuestas surgió:

- Los ciudadanos que manifestaban que no es $t$ á $n$ dispuestos a sumir mayores costes en sus compras eligiendo empresas con un mejor comportamiento social y medioambiental, representaban algo más de uno de cada cinco $(22,9 \%)$, porcentaje inferior en dos puntos a otra investigación de hacía dos años (año 2008),

- Sin embargo, casi seis de cada diez (59,5\%), si tuvieran la información, hubieran elegido una de esas empresas y pudiendo llegar a pagar hasta un 10\% más por sus adquisiciones, siendo el porcentaje de quienes manifiestan esa intención superior en algo más de siete puntos al que se registraba en 2008.

- Fueron sensiblemente inferiores los porcentajes de quienes se muestran dispuestos a asumir mayores costes por la elección: $10,5 \%$ los que asumirían un sobre coste de entre el $11 \%$ y el $25 \%$ y un $3,0 \%$ los que asumirían un incremento de entre el $26 \%$ y el $50 \%$. Los porcentajes de quienes en 2008 se habían manifestado dispuestos a esos dos mismos comportamientos eran superiores en dos y un punto y medio respectivamente.

Por otra parte, al momento de analizar la RSE, los atributos sociales juegan un papel importante en determinar las intenciones de compra del consumidor, incluso en presencia de otros atributos intangibles como la marca y el país de origen, es así como: (1) los atributos sociales son por lo general más influyente en los países desarrollados que en los mercados emergentes, (2) atributos sociales son influyentes para productos de baja y alta participación y para condiciones ambientales y laborales, y (3) el conocimiento previo de los atributos sociales tienden a ser un predictor fuerte de influencia sobre las intenciones de compra que los atributos intangibles (Auger P. et al., 2010).

Otra investigación que es de destacar fue la realizada por Marquina Feldman P. \& Reficco E. (2012) sobre disposición a pagar por RSE de consumidores bogotanos, la misma evidencia la existencia de una relación significativa de los atributos de responsabilidad social 
empresarial y de las competencias corporativas sobre el comportamiento de compra del consumidor de la ciudad de Bogotá. A su vez, los resultados muestran que la probabilidad de compra de los consumidores de zapatillas aumenta si la empresa fabricante cumple con determinadas características en su gestión: compromiso con el medio ambiente, buen trato a los trabajadores, apoyo a programas de lucha contra la pobreza, calidad en los productos, innovación tecnológica; por el contrario, la probabilidad de compra disminuiría si la empresa en cuestión fuese líder en su sector. Es así como este estudio ha mostrado también que los consumidores están dispuestos a pagar una mayor proporción del precio del producto por atributos empresariales vinculados a la responsabilidad social, tomados en conjunto, en comparación a los atributos de competencias corporativas ( $39 \%$ vs $18 \%$, respectivamente), si bien la calidad de los productos como atributo individual genera una disposición a pagar mayor que la del resto de atributos analizados, según nuestro resultados. La proporción en cuestión, es de poco más de 2 a 1 a favor de los atributos de RSE. En ese sentido, la responsabilidad social puede constituirse en un factor diferenciador para los consumidores y por lo tanto le puede agregar valor a aquellas empresas que logren ser percibidos favorablemente respecto de esta variable por parte de sus potenciales compradores.

\section{Objetivo de la investigación}

El presente trabajo tiene como objetivo general: "Determinar la disposición a pagar (DAP) de los consumidores en el Gran La Plata por atributos de Responsabilidad Social Empresaria".

A su vez como objetivos específicos:

1. Determinar si los consumidores están dispuestos a pagar por atributos de Responsabilidad Social Empresaria.

2. Determinar si el género, edad y el nivel educativo universitario completo está relacionado con la disposición a pagar por atributos de Responsabilidad Social Empresaria.

Cabe aclarar que los atributos de RSE seleccionados para la investigación fueron:

- "Prácticas Laborales" (satisfacción de las personas por la tarea realizada, la oportunidad de interrelación con otras personas, el desarrollo de capacidades y habilidades);
- "Protección Medio Ambiente" (uso de productos biodegradables, prevención contaminación);

- "Participación Activa y Desarrollo de la Comunidad" (inversiones socialmente responsables en la comunidad local, creación de empleo);

A su vez para determinar la disposición a pagar, primero se hizo una prueba piloto con tres productos de primera necesidad: leche, zapatillas y medicamentos, del cual surgió que el producto que mejor se adaptó al tipo de cuestionario fueron las zapatillas, por otra parte dado que es un producto ampliamente comprado por la población objeto del estudio, es que se decidió determinar la disposición a pagar de las mismas.

\section{Metodología}

El método seleccionado fue el de valoración contingente, a través del mismo se simuló un mercado mediante un cuestionario, en el cual el encuestador realizó el papel de oferente del bien y el encuestado de demandante.

La muestra fue seleccionada de forma aleatoria en distintos barrios del Gran La Plata (Provincia de Buenos Aires, Argentina).

Cabe aclarar que debido al requerimiento de un cierto nivel de procesamiento de información, se seleccionarán a aquellos grupos etáreos mayores a 18 años de edad y con nivel educativo igual o superior a secundario completo.

Según el Censo Nacional de Población, Hogares y Viviendas 2010, publicado por el Instituto Nacional de Estadísticas y Censos (INDEC), la población mayor de 18 años del Gran La Plata asciende a 469.699 habitantes $(\mathrm{N})$.

Para el cálculo del tamaño de la muestra, se considero un nivel de confianza (Z), del $95 \%$ y un error (e) del $3 \%$ (especificamente $2,63 \%$ ). Respecto a p y q (porcentajes de estimación), en este caso, al no existir estadísticos de referencia, se ha optado por el $50 \%$ para cada estimación, de esta manera se obtuvo una muestra de $n=310$.

De esta manera, del diseño elegido surge un estudio transversal ya que se observan las características y opiniones de la población objeto de estudio, en un solo momento temporal, es decir, la investigación se centro en el "hoy" y no pretendió relevar el comportamiento de las variables en el tiempo. 
El relevamiento se llevó a cabo mediante una encuesta. El cuestionario fue estructurado en 3 bloques siguiendo los lineamientos y procedimientos aconsejados en la bibliografía consultada para la determinación de la disposición a pagar por el método de valoración contingente, es así como el cuestionario contó con 3 bloques:

1. Características socioeconómicas del encuestado, sexo, edad y nivel educativo.

2. Conducta y percepciones del consumidor acerca de la responsabilidad social empresaria; datos sobre las preferencias de los consumidores en relación a los distintos atributos de la responsabilidad social empresaria seleccionados: Prácticas Laborales, Protección Medio Ambiente y Participación Activa y Desarrollo de la Comunidad.

3. Disposición a pagar por los atributos de responsabilidad social empresaria seleccionados, mencionados en el punto anterior.

Para analizar la disposición a pagar por los atributos de RSE, se procedió a la estimar, un modelo logit binario (para cada uno de los atributos), con el que se obtendrá una función que permita clasificar a los encuestados en uno de los dos grupos establecidos por la variable dependiente dicotómica o binaria: 0 los que no están dispuestos a pagar y 1 los que sí lo están. Las variables independientes serán las siguientes: género, edad y estudios universitarios completos, las mismas se detallan en la tabla 1.

\begin{tabular}{|c|c|c|}
\hline $\begin{array}{l}\text { Nombre de las variable generadas } \\
\text { (independientes o predictoras) }\end{array}$ & Tipo de Variable & Valores asignados \\
\hline Edad & $\begin{array}{l}\text { Continua medida en } \\
\text { años }\end{array}$ & Valores entre 18 y 90 \\
\hline \multirow{2}{*}{ Género } & \multirow{2}{*}{ Binaria } & Masculino = 1 \\
\hline & & Femenino $=0$ \\
\hline Universitario Completo & Binaria & $\begin{array}{l}\text { Nivel educativo univeritario } \\
\text { completo o superior }=1 \\
\text { Nivel educativo universitario } \\
\text { incompleto o menor }=0\end{array}$ \\
\hline
\end{tabular}

De esta manera dada la variable dependiente y las variables predictoras, las hipótesis del modelo serán las siguientes:

Ho: La Disposición a pagar por atributos de RSE no puede ser explicada a través por la edad, género y el nivel educativo universitario completo.

Por otra parte, para la determinación del monto de la DAP, se estimará un modelo de regresión, donde las variables independientes serán nuevamente las vari- ables detalladas en la Tabla I (género, edad, universitario completo) y la variable dependiente es el porcentaje adicional al precio actual que cada uno de los encuestados estaría dispuesto a pagar, por tal motivo es que el valor oscila entre 0 y 100. La estimación de la función, se realizará mediante un modelo de regresión multivariante censurado en cero, para lo cual se utilizará el modelo tobit (o modelo de regresión censurado) dada la distribución de la DAP, con una acumulación de observaciones en el valor cero (los ceros reales), la imposibilidad de tener valores negativos.

\section{Resultados de la Investigación}

En primer lugar, en cuanto a las características de los encuestados, se obtuvieron iguales proporciones de hombres y mujeres, respecto a la edad, el 45,5\% tenía una edad entre 26 y 35 años, el $18,1 \%$ entre 36 y 45 años y un $13,5 \%$ entre 46 y 55 años. En cuanto al máximo nivel educativo, el 57,1\% tenía nivel universitario completo o superior.

Analizando la importancia asignada por los encuestados a la RSE, como se puede observar en el Gráfico 1, al momento de comprar un producto un $71,90 \%$ de los encuestados manifestaron darle mucha o moderada importancia a la RSE de las empresas, mientras que el $28,4 \%$ lo considera poco importante, seguido de un $9,7 \%$ que no le asigna ningún tipo de importancia.

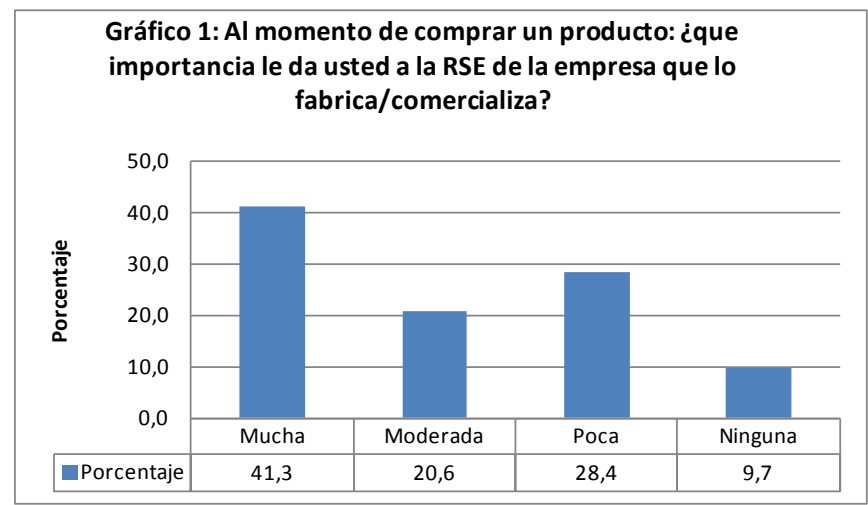

Analizando la disposición a pagar un \% adicional al actual precio que los encuestados pagarían por atributos de RSE, en el gráfico 2 podemos observar que para el atributo de RSE "Prácticas Laborales" un 52,9\% no estaría dispuesto a pagar un \% adicional, esto se revierte para el atributo "Participación Activa y Desarrollo de la Comunidad" estando dispuestos a pagar un 59\%, por último este porcentaje se incrementa significativamente para el atributo "Protección del Medio Ambiente" ya que un $76,5 \%$ estaría dispuesto a pagar un $\%$ adicional al actual precio. 


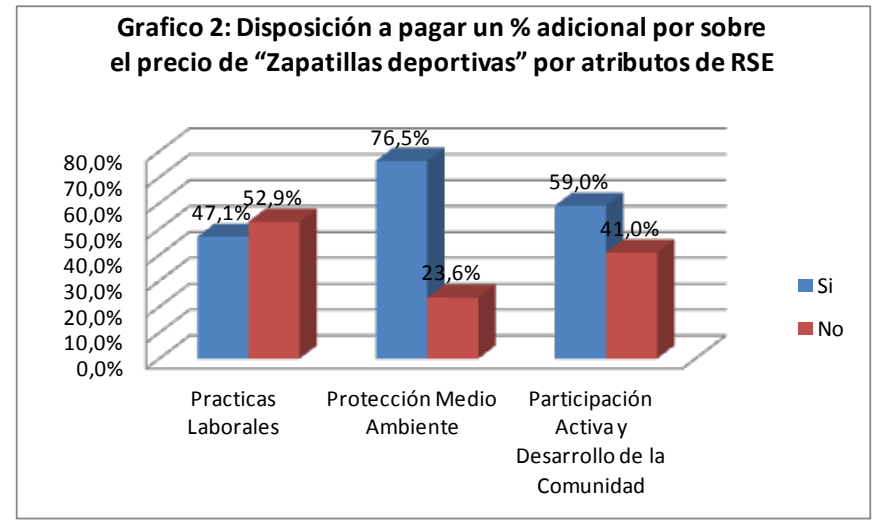

Por otra parte, para analizar si el género, edad y nivel educativo universitario completo, influye sobre la decisión de pagar o no, de acuerdo a lo explicado en la metodología, se procedió a estimar un modelo de logit binario para cada uno de los atributos analizados (Tabla 2).

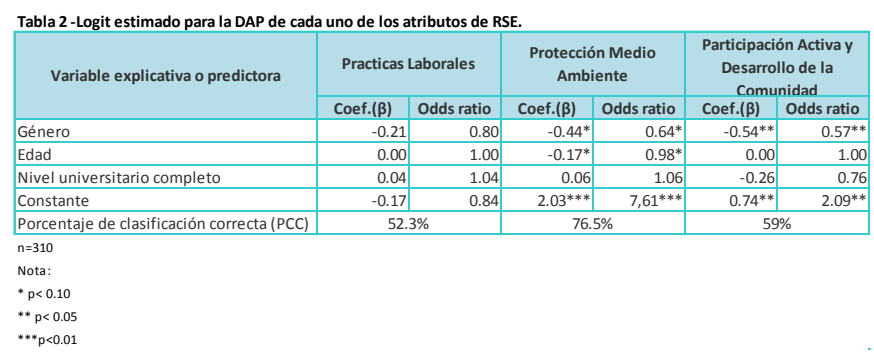

De los análisis logit podemos destacar:

- Para la DAP por el atributo de RSE "Prácticas Laborales", ninguna de las variables resultó significativa ( $p$-valor $<0.10)$, por tal motivo podemos concluir que el género, la edad y el contar con nivel universitario completo no influye en la disposición a pagar por el mencionado atributo de RSE.

- Para la DAP por el atributo de RSE Protección del Medio Ambiente, las variables género y edad resultaron significativas, a diferencia de la variable nivel universitario completo que no lo resultó. De la interpretación de los odds ratios, podemos mencionar que en el caso de ser hombre la probabilidad de estar dispuesto a pagar por la Protección del Medio Ambiente, disminuye 0.64 veces, siendo la variación porcentual de la probabilidad de -36 \%. La misma relación inversa presenta la variable edad, de esta manera a mayor edad disminuye la probabilidad de estar dispuesto a pagar por el mencionado atributo.

- En cuanto al logit estimado para la DAP por el atributo de RSE Participación Activa y Desarrollo de la Comunidad, sólo la variable género resultó significativa. En cuanto al género, como en la anterior logit, la variable presenta una relación inversa con la variable dependiente, es decir, el ser hombre disminuye la probabilidad de una DAP positiva.
Por último se buscó dar respuesta al siguiente interrogante: ¿El monto de la disposición a pagar (DAP) por atributos de RSE, está relacionado con el género, la edad y el nivel educativo universitario completo?

Para dar respuesta a este interrogante a continuación se presentan 3 modelos tobit, uno para cada de los atributos de RSE planteados (Tabla 3). Del análisis de los mismos podemos destacar:

- Analizando el monto de la DAP del atributo Prácticas Laborales, se puede observar que en el modelo planteado sólo resulto significativa la variable género $(p$-valor $<0.10)$, presentando una relación inversa con la variable dependiente, es decir, en el caso de ser hombre el monto de la DAP por el mencionado atributo, disminuiría.

-Por otra parte, en cambio en el segundo modelo tobit que se presenta, resultan significativas con un $p$-valor $<0.01$ tanto las variables género y edad, presentando ambas variables una relación negativa con el monto de la disposición a pagar por el atributo de RSE "Protección del Medio Ambiente", esto significativa que el monto de la DAP disminuye en caso de ser hombre y a medida que aumenta la edad.

- Por último, en el tercer modelo tobit propuesto, sólo resulta significativa la variable género, presentando nuevamente una relación inversa con la variable dependiente, en este caso el monto de la DAP por el atributo de RSE Participación activa y desarrollo de la comunidad.

\begin{tabular}{|c|c|c|c|}
\hline \multirow[t]{2}{*}{ Variable explicativa o predictora } & Practicas Laborales & $\begin{array}{l}\text { Protección Medio } \\
\text { Ambiente }\end{array}$ & $\begin{array}{l}\text { Participación Activa y } \\
\text { Desarrollo de la Comunidad }\end{array}$ \\
\hline & Coef.(B) & Coef.( $(\beta)$ & Coef.(B) \\
\hline Género & -5.26 & -10.86 & -9.77 \\
\hline Edad & 0.00 & -0.33 & -0.58 \\
\hline Nivel universitario completo & -4.17 & -3.54 & -4.21 \\
\hline Constante & 0.83 & 30.52 & 11.51 \\
\hline$n=$ & 310 & 310 & 310 \\
\hline Obs. Censuradas en cero $=$ & 164 & 73 & 127 \\
\hline $\begin{array}{l}\text { Contraste de razón de } \\
\text { verosimilitudes }\end{array}$ & $x^{2}(3)=3.89(0.27)$ & $x 2(3)=24.69(0.00)$ & $x 2(3)=15.32(0.00)$ \\
\hline
\end{tabular}

\section{Conclusiones}

Cuando se les preguntó a los encuestados acerca de la importancia que le daban al momento de la compra de un producto a la RSE de la empresa fabricante y/o comercializadora, un $41.3 \%$ manifestó considerarla muy importante, de esta manera se puede considerar que el trabajo ha puesto de manifiesto un importante grado de concientización sobre la RSE.

En cuanto a la disposición a pagar un \% adicional al actual precio, el atributo de RSE Protección del Medio Ambiente, es el que mayor cantidad de resultados positivos, el $76.5 \%$ de los encuestados estaría dispuesto 
a pagar un \% adicional. Estos resultados son esperables teniendo en cuenta que, en general, la sociedad suele tener una alta sensibilidad hacia los problemas ambientales. En cambio si analizamos el atributo de RSE Practicas Laborales la disposición de pagar un $\%$ adicional al actual precio el porcentaje disminuye a un $47,1 \%$.

Si se compara el porcentaje de los encuestados que le dio mucha o moderada importancia a la RSE resulta ser el $71,9 \%$, si comparamos esto con el porcentaje que Sí estaría dispuesto a pagar por RSE, en el caso de la Protección al Medio Ambiente el porcentaje es superior, en cambio en los otros dos casos: Prácticas Laborales y Participación Activa y Desarrollo de la Comunidad los porcentaje son bastante inferiores (47.1\% y $59 \%$, respectivamente), esta diferencia entre importancia asignada y disposición de pago, podría deberse, por alguna manifestaciones efectuadas verbalmente por los encuestados, a que algunos consideran que el problema es competencia de la administración pública, no creen apropiado un incremento del precio para sufragar este tipo de atributos (por ejemplo, el de Prácticas Laborales). No obstante queda para futuras investigaciones el analizar los motivos de estas diferencias.

Cabe aclarar que para la DAP positiva en el atributo Protección del Medio Ambiente, en caso de ser hombre y tener mayor edad disminuye la probabilidad de una DAP positiva, esto deja de manifiesto mayor sensibilización de las mujeres y los más jóvenes frente al tema. Respecto a la DAP Participación Activa y Desarrollo de la Comunidad, la misma también resultó tener una relación negativa con la variable género, al ser hombre disminuye la probabilidad de una DAP positiva. Estas mismas relaciones se presentaron al analizar el monto de la disposición a pagar, cabe resaltar que en este caso para los tres atributos resultó significativa la variable género, con una relación inversa con las tres variables dependientes, es decir, para cualquiera de los tres atributos, en el caso de ser hombre el monto de las respectivas DAP disminuye.

Es de destacar como la disposición adicional al pago atributos de RSE calculada en este trabajo, pese a que venga dada en un mercado hipotético y, por tanto, muestre un compromiso también hipotético, es reflejo de una aceptación mayoritaria a asumir un precio adicional por RSE.

Este estudio también da lugar a una serie de oportunidades para futuras investigaciones. En primer lugar, la metodología podría ser aplicada a consumidores de distintas regiones, así como también se podría replicar el análisis a otros productos comercializados en el mercado, con el fin de realizar estudios compara- tivos. Por otra parte, de todos los resultados expuestos surge la oportunidad que se les presenta a las empresas por un lado de desarrollar nuevos productos socialmente responsables y por otro lado reforzar su imagen de marca destacando los atributos de RSE que actualmente presentan, puesto que como se observó los mismos son vistos en general positivamente por los consumidores. Por último, se deja planteada para futuras investigaciones una investigación adicional que examine la rentabilidad de las prácticas de RSE sobra la inversión requerida.

\section{Bibliografía}

ARREDONDO TRAPERO, F., MALDONADO DE LOZADA, V. y DE LA GARZA GARCÍA, J.(2010). "Consumers and their buying decision making based on price and information about corporate social responsibility (csr). Case study: undergraduate students from a private university in Mexico" Estudios Gerenciales, 26 (117), pp. 103-117. Universidad ICESI Cali, Colombia. Disponible en: http://www.redalyc. org/src/inicio/ArtPdfRed.jsp?iCve=21218551005

ARGANDOÑA A. (2008). "¿Es cara la responsabilidad social?". IESE Business School. Universidad de Navarra.

AUGER P., DEVINNEY T., LOUVIERE J. and BURKE P. (2010). "The importance of social product attributes in consumer purchasing decisions: A multi-country comparative study". International Business Review , 19, pp. 140-159.

CONFEDERACIÓN DE CONSUMIDORES Y USUARIOS (CECU) RSE (2010). "La opinión y valoración de los consumidores sobre la Responsabilidad Social de la Empresa en España". Ediciones y Marketing, S.L. (EDIMARK).

DESCUBRIENDO ISO 26000. (2010). Organización Internacional de Estandarización.

DU, S., BHATTACHARYA, CB and SEN, S. (2010). "Corporate Social Responsibility and Competitive Advantage: Overcoming the Trust Barrier" ESMT Working Paper 10-006. Available at SSRN: http:// ssrn.com/abstract $=1690423$

DU, S., BHATTACHARYA, CB and SEN, S. (2007). "Reaping Relational Rewards from Corporate Social Responsibility: The Role of Competitive Positioning International" Journal of Research in Marketing 24, pp. 224-241. Available at SSRN: http://ssrn.com/abstract $=2333555$ 
FERNÁNDEZ DE CASTRO RIVERA J. (2008). "Ensayo sobre RSE" Foment del Treball Nacional, 2008. La RSC y la maximización conjunta de beneficios. Universidad de Barcelona.

FERNÁNDEZ KRANZ, D. and MERINO CASTELLÓ, A. (2005). "¿Existe disponibilidad por pagar por responsabilidad social corporativa?, Percepción de los consumidores". Universia Business Review- Actualidad Económica- Tercer Trimestre.

GILLI J. (2011). Ética y Empresa. Ediciones Granica S.A. Buenos Aires, Argentina.

KRENG B. and HUANG M. (2011). "Corporate Social Responsibility: Consumer behavior, corporate strategy and public policy". Social Behavior and Personality, 39, pp. 529-542.

LOZANO (2003). "Obstáculos para la Responsabilidad Social Corporativa". La Bolsa.com [Europa Press]. Disponible en http://www.labolsa.com/canales/781.
MARTÍNEZ CERNA L (2006). 2¿Una empresa socialmente responsable es más competitiva?" Tercer Encuentro Internacional sobre Economía, Política y Ética. España.

MARQUINA FELDMAN P. y REFICCO E. (2012) "Impacto de la Responsabilidad Social Empresarial en Comportamiento de Compra y Disposición a Pagar de Consumidores Bogotanos". CENTRUM Católica's Working Paper Series No. 2012-09-0014 / September 2012 - Pontificia Universidad Católica del Perú.

NORMA INTERNACIONAL ISO 26000 (2010), Primera edición.Versión española, publicada en Suiza.

PORTER, M. E., and KRAMER, M. R. (2006). "The link between competitive advantage and corporate social responsibility". Harvard business review, 84(12), 78-92. 